\title{
VALIDATION OF DYNAMIC GB TRANSMISSION SYSTEM MODELS WITH PMU DATA FOR HARDWARE-IN-THE-LOOP STUDIES Peter Imris $^{1}$, Martin Bradley ${ }^{1}$, Gareth Taylor ${ }^{*}$, Yun Li $^{2}$
}

${ }^{1}$ Department of Electronic and Computer Engineering, Brunel University London, Uxbridge, UB8 3PH, UK

${ }^{2}$ National Grid Electricity System Operator, St. Catherines Lodge, Wokingham, RG41 5BN, UK

Peter.Imris@brunel.ac.uk, Martin.Bradley@brunel.ac.uk, Gareth.Taylor@brunel.ac.uk,

Yun.Li@nationalgrideso.com

\begin{abstract}
Keywords: FAST FREQUENCY PHENOMENA, GREAT BRITAIN TRANSMISSION SYSTEM, HARDWARE-IN-THE-LOOP, LOSS OF MAINS.
\end{abstract}

\begin{abstract}
Decarbonisation of the electrical power system in Great Britain (GB) has impacted the dynamic behaviour of the power system, not least due to the reduction in system inertia. Following disturbance events on the power system, Fast Frequency Phenomena (F2P) can be observed which may cause mal-operation of Loss-of-Mains (LoM) protection relays on distributed generation where the relays use Rate of Change of Frequency (RoCoF) or Vector Shift (VS) to detect loss of mains. The research presented in this paper aims to validate a full model of the GB transmission system using Phasor Measurement Unit (PMU) data from system events. The model is implemented in the DIgSILENT PowerFactory analysis software. Three types of F2P are compared in the period following the disturbance when LoM protection may be affected: simulation of electromagnetic (VS) phenomena shows some differences to actual PMU measurements, but the electromechanical and mechanical phenomena (affecting RoCoF) are in good agreement. The distribution of inter-area oscillation frequency modes following the disturbances are also closely represented in the model. Based on these studies, it can be concluded that the model is suitable for F2P studies using Hardwarein-the-Loop simulation with real LoM protection relays.
\end{abstract}

\section{Introduction}

The composition of electricity generation has changed significantly in recent years across the world [1] due to the growth in renewables and, for Great Britain (GB), HVDC interconnections; these have been displacing traditional generation sources such as coal. This trend is expected to continue in coming years in order to meet environmental targets and legislation. As a consequence, the inertia and fault levels on the GB system have decreased such that disturbances in the power system, e.g. line or generator tripping, can lead to larger frequency fluctuations across wider geographical areas. These include a larger Rate of Change of Frequency (RoCoF) and/or increase in Vector shift during the faults. If unmitigated, these fluctuations may exhibit similar behavior to that which is present during system islanding and thus may lead to the malfunction of Loss-of-Mains (LoM) protection relays [2]. This phenomenon was observed on the GB transmission system during the incident on $9^{\text {th }}$ August 2019, when approximately $500 \mathrm{MW}$ of renewable generation tripped from maloperation of either RoCoF or VS protection following losses of transmission-connected generation. The combined loss of generation led to the operation of Low Frequency Demand Disconnection relays at $48.8 \mathrm{~Hz}$ resulting in the disconnection of approximately 1 million customers [3].

This paper is a post-print of a paper submitted to and accepted for publication in Conference Proceedings of the 15th International Conference on Developments in Power System Protection (DPSP 2020) and is subject to Institution of Engineering and Technology Copyright. The copy of record is available at IET Digital Library.
A research project at Brunel University London sponsored by the National Grid Electricity System Operator (NGESO) aims to investigate the Fast Frequency Phenomena (F2P) which occur during system disturbances. A special interest is in the mal-operation of LoM protection under the new conditions of reduced inertia and fault levels on the power system. In order to investigate these $\mathrm{F} 2 \mathrm{P}$, the project includes three main objectives: (1) Visualization of the F2P recorded by Phasor Measurement Units (PMUs) [4], (2) validating the ability of GB system models and analysis software to reproduce these phenomena and (3) using these models for Hardware-in-theLoop (HIL) studies with the OPAL-RT real time digital simulator. The HIL studies involve stress-testing real LoM relays in current and future inertia scenarios.

The research conducted in this paper contributes to the second objective: validation of models \& modelling tools. Accurate modelling will be essential to any new operational planning processes aiming to forecast the geographic variation in ROCOF and Vector Shift for credible faults; such new processes may be able to deliver secure operation at lower cost. Accurate modelling will also contribute to Objective 3 , by giving confidence that HIL studies are reflective of real ROCOF and VS phenomena. 


\section{Great Britain transmission system and models}

\subsection{Description of the GB transmission system}

The GB transmission system is an isolated synchronous system, with only HVDC connections to continental Europe and Ireland, as illustrated in Figure 1. The GB system currently faces challenges arising from low system inertia due to growing levels of renewables and HVDC integration.

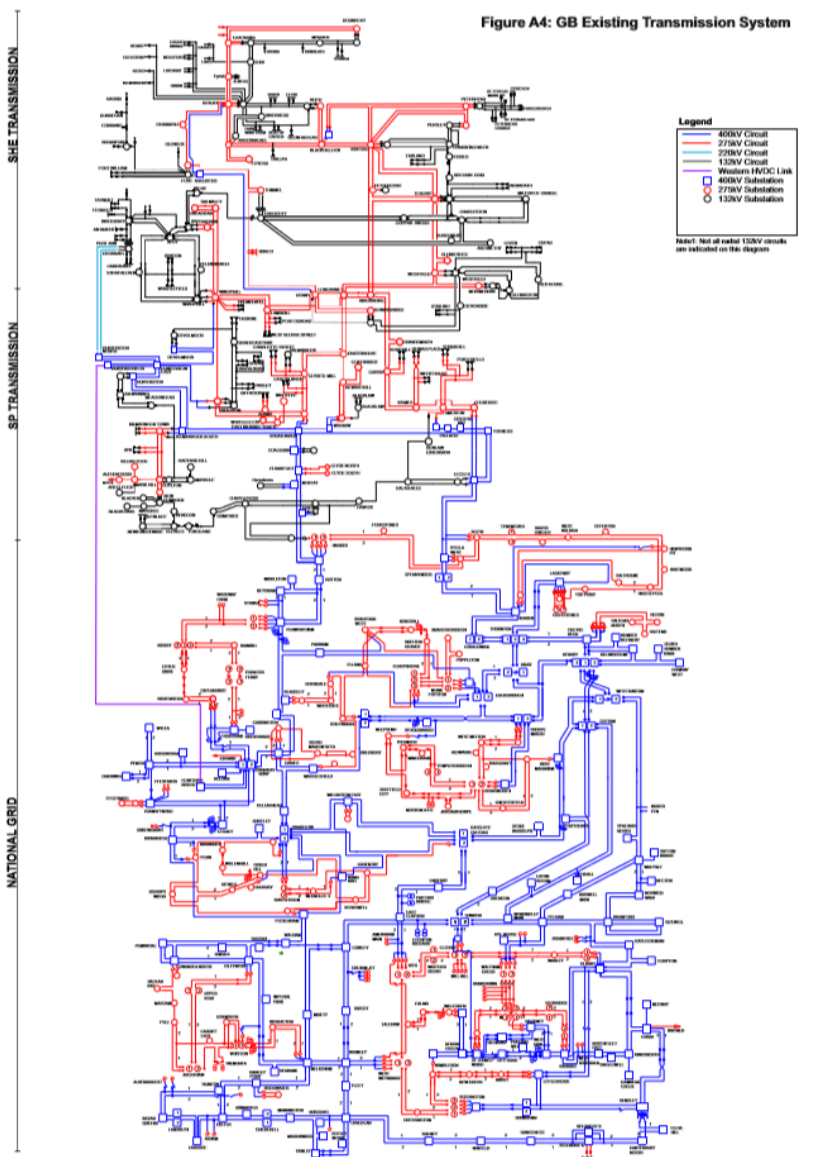

Figure 1 The Schematic of GB transmission system, 2018, [5].

\subsection{Phasor Measurement Units on the GB system}

Approximately 90 PMUs or Waveform Measurement Units (WMUs) are currently installed across the GB transmission system to measure parameters such as voltage and frequency, with GPS synchronisation. The associated infrastructure was built prior to the current project, and is described in the project "VISOR": "Visualisation of Real Time System Dynamics using Enhanced Monitoring", [6]. Figure 2 shows the location of the PMUs, WMUs, Data Centres, Data Hub and communications. The project in this paper used the data obtained from these PMUs to validate the models under the consideration. The IEEE C37.118.1 standard on PMUs defines how frequency is estimated from a derivative of the positive sequence voltage angle, as shown in Figure 3 [7]. Based on this principle, a standard PMU will correctly interpret frequency under slowly-changing conditions; however, fast electromagnetic (Vector Shift) phenomena may be misreported as a change in frequency, [2], [4].

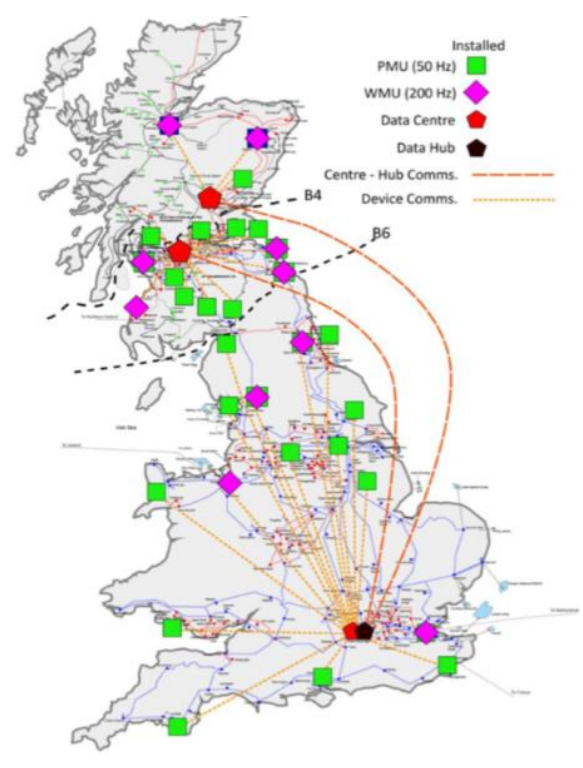

Figure 2 The WMU and PMU infrastructure, (VISOR) [6].

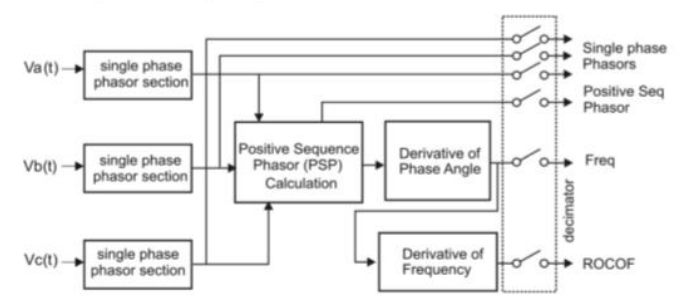

Figure 3 A PMU model using the positive sequence phasor. IEEE Standard C37.118.1 ${ }^{\text {TM}}-2011$ [7].

\subsection{Models of the GB transmission system}

NGESO has developed two models of the GB transmission system. A full model of the system is used for Off-Line Transmission Analysis and is referred to as the "OLTA" model. This model is used for design, planning and optimization studies from 7 years ahead down to hours ahead in the control room. Amongst other things it is able to monitor and predict system dynamics to efficiently facilitate increased levels of renewable generation, [8]. The transmission circuits in the OLTA model are presented in Figure 1, [5] (substation details and distribution circuits are not included).

NGESO has also developed also a reduced model of the GB system to permit faster investigation of future scenarios and also for external research use [9], but this paper focuses on the full OLTA model validation.

\section{Fast Frequency Phenomena (F2P) in the GB transmission system}

\subsection{Types of recorded Fast Frequency Phenomena}

Based on observed PMU measurements and taking into account their frequency calculation algorithm, F2P cannot be understood as a single type of phenomenon only. Immediately following a disturbance, there is an electromagnetic Vector 
Shift effect, whereby power flows and voltage angles adjust very quickly (e.g. within $200 \mathrm{~ms}$ ). Next there are slower but longer-lasting electromechanical phenomena, arising from local and inter-area oscillation modes that have been excited by the disturbance (the "AC" component of measured RoCoF response). Finally, there is the simple mechanical mode of response, where the average system-wide frequency responds to any imbalance in supply and demand (the "DC" component of measured RoCoF response). All three types of phenomena have the potential to trigger maloperation of RoCoF relays, and in addition the electromagnetic phenomenon has the potential to trigger maloperation of Vector Shift protection. The electromagnetic phenomenon will be governed by the electrical network and sub-transient (fault level) characteristics of generators, whilst the other two phenomena (electromechanical and mechanical) will be governed by generation controllers and inertia.

\subsection{Impact of F2P on Loss-of-Mains protection}

LoM protection is designed to safely disconnect embedded generation following islanding from the main power system, by detecting significant RoCoF or Vector Shift. However, the threshold values for these parameters given in the original UK Engineering Recommendation G59 $(0.125 \mathrm{~Hz} / \mathrm{s}$ and 6 degrees respectively) may now be exceeded during a main system disturbance that does not involve islanding (e.g. the trip of a generator or HVDC interconnector or a correctly cleared transmission fault). This could add to the original disturbance and potentially lead to cascade tripping. In 2018 the G59 RoCoF limit for new connections was increased to $1 \mathrm{~Hz} / \mathrm{s}$ and Vector Shift was no longer approved; an industry initiative was also launched to update existing LoM protection to the new settings, but this will take some years and may not achieve full coverage of all embedded generation installations, so there will continue to be a residual risk of maloperation.

\section{Model validation with PMU data}

\subsection{Disturbance event for validation}

For validation of the full OLTA model of the GB system, a typical transmission disturbance was selected: the trip of an HVDC link connected in the South-East of the system. In this event, shown in Figure 4 and Figure 5, one pole of the HVDC link tripped at $\mathrm{t}=20 \mathrm{~s}$ while importing $500 \mathrm{MW}$, and the system frequency fell for about 10s after the event occurred. Three phases of system behaviour can be identified following this event. The first is a rapid redistribution of power flows and voltage angles (Vector Shift) around the network, governed by electromagnetic effects. This is reported by the PMUs as a high RoCoF transient (e.g. $-1.8 \mathrm{~Hz} / \mathrm{s}$ ) and sudden dip in frequency within the first $200 \mathrm{~ms}$. The second phase comprises local and inter-area electromechanical oscillations of $1.5 \mathrm{~Hz}$ and 0.5 $0.6 \mathrm{~Hz}$ respectively (the "AC" electromechanical component). The third phase involves a common-mode "DC" component governed by the overall power imbalance of the system. This DC RoCoF starts at $-0.045 \mathrm{~Hz} / \mathrm{s}$ and in 10 seconds falls to zero $\mathrm{Hz} / \mathrm{s}$. Figure 4 shows data from all PMUs while Figure 5 gives more detail from just one PMU.

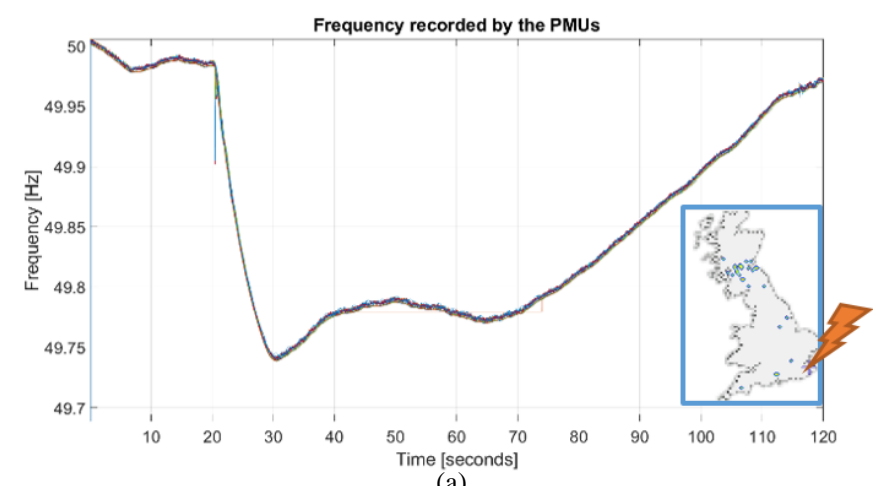

(a)

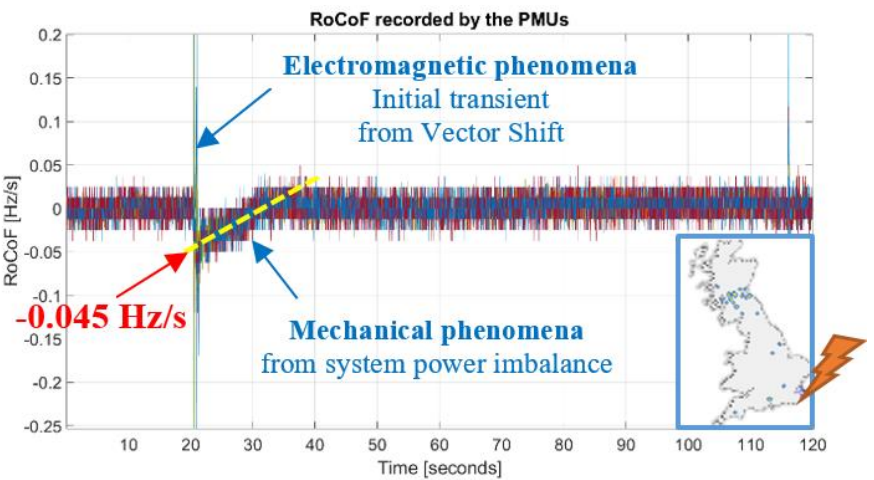

(b)

Figure 4 Data from all PMUs during the HVDC trip event 20.4.2018. The disturbance location is shown on the map with a red arrow. (a) the Frequency recorded by all PMUs, (b) the RoCoF recorded by all PMUs.

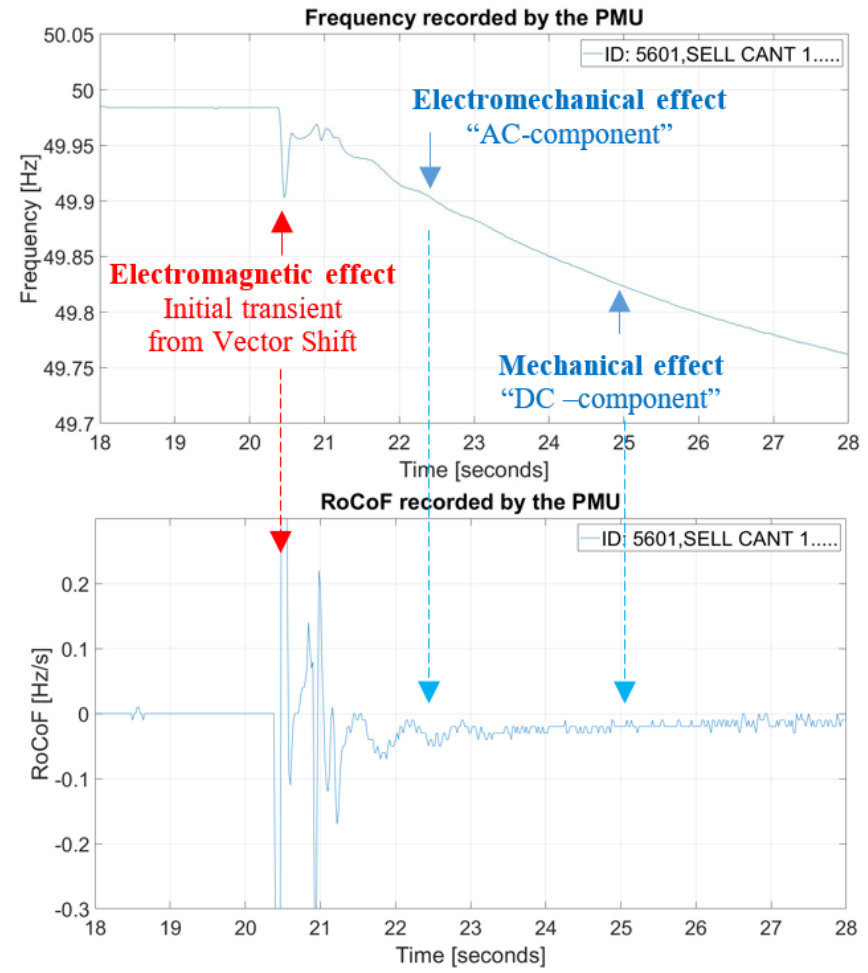

Figure 5 Three types of F2P investigated for validation purposes. Data is from the substation where the HVDC trip occurred. 


\subsection{Model updates}

For model validation, the full model of the GB transmission system was updated based on system conditions at the time of the event. The electromagnetic and electromechanical phenomena were then investigated separately.

\subsection{Investigation of Electromechanical and Mechanical Phenomena}

Figure 6, Figure 7 and Figure 8 give the comparison of PMU and OLTA study data, and show that the general frequency behaviour during the disturbance (arising from the electromechanical and mechanical behaviour of the generator's rotors) is well represented by the full GB model. The comparison results are from three locations in the network: Sellindge (in the South-East of the transmission system), Staythorpe (Central) and Dalmally (North). It can be seen from the time and frequency domain comparisons that the "DC" component of the RoCoF as well as the "AC" oscillations show good correlation. Such a good correlation is of course expected as the model and the analysis software are intended to provide accurate representation of these phenomena.

Figure 9 shows the comparison of dominant frequency modes on a geographic basis. The $0.67 \mathrm{~Hz}$ mode in the North is an inter-area oscillation, whilst the $1.3 / 1.4 \mathrm{~Hz}$ mode in the South is a local oscillation mode.

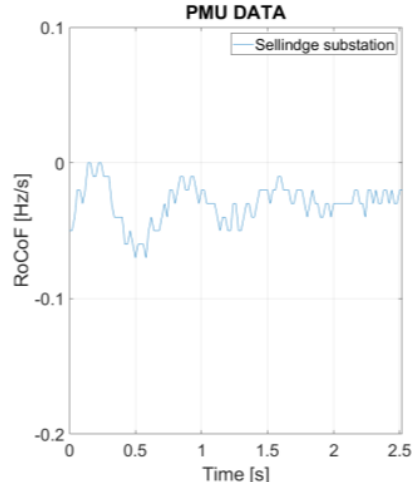

(a)

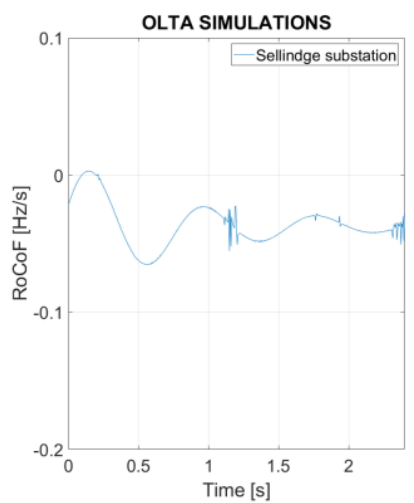

(c)

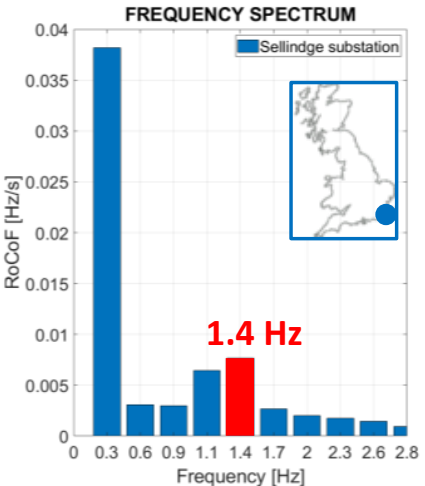

(b)

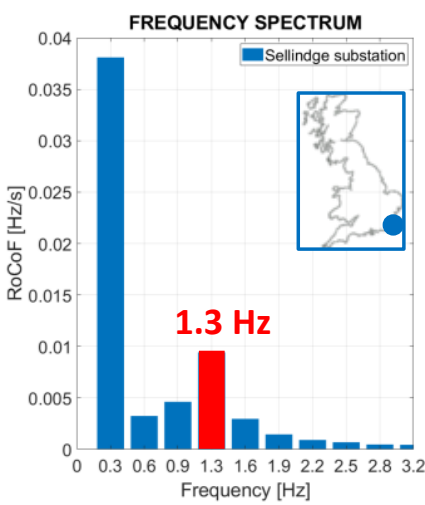

(d)
Figure 6: Comparison of F2P in time and frequency domains at Sellindge substation; PMU data ( $\mathrm{a}$ and $\mathrm{b}$ ) and OLTA simulation (c and d).

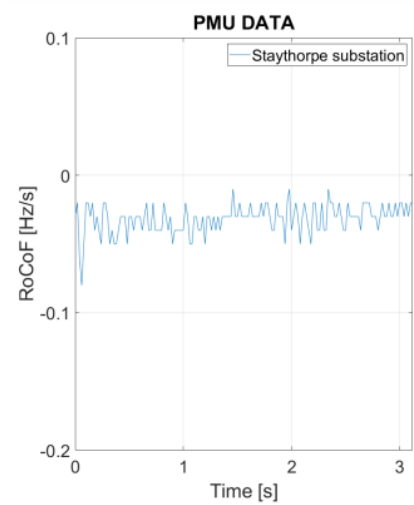

(a)

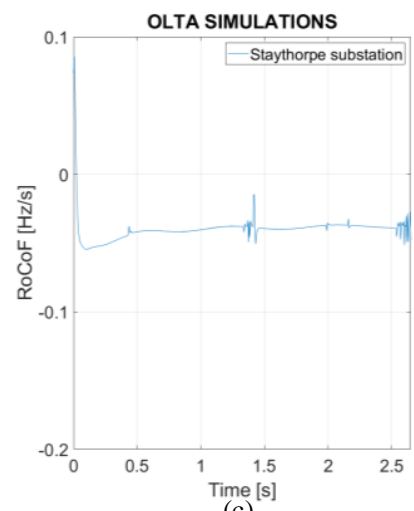

(c)

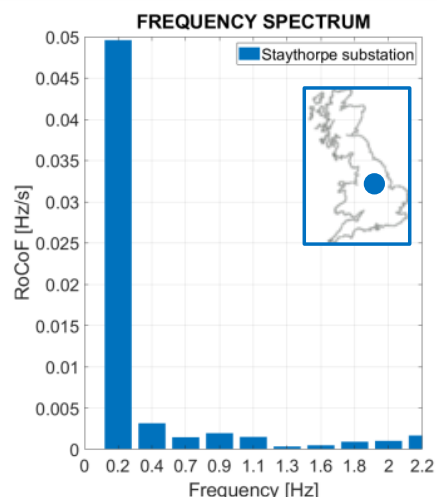

(b)

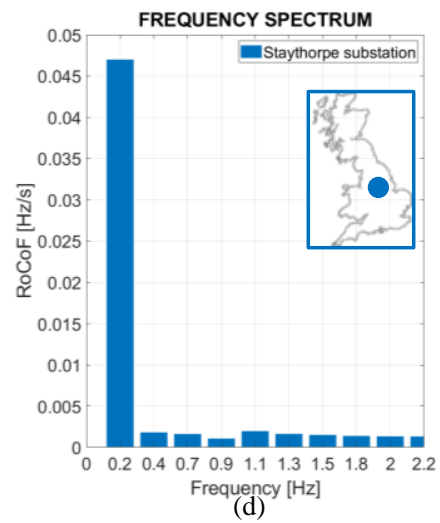

Figure 7: Comparison of F2P in time and frequency domains at Staythorpe substation; PMU data ( $\mathrm{a}$ and $\mathrm{b}$ ) and OLTA simulation (c and $\mathrm{d}$ ).
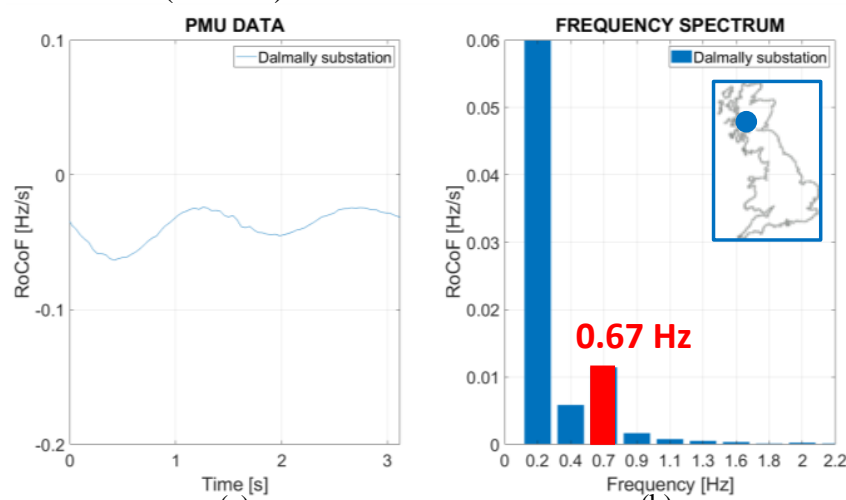

(a)

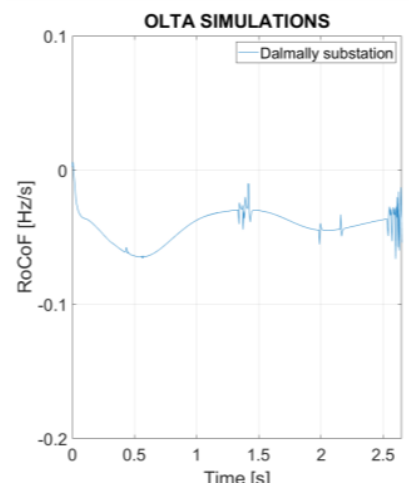

(c)

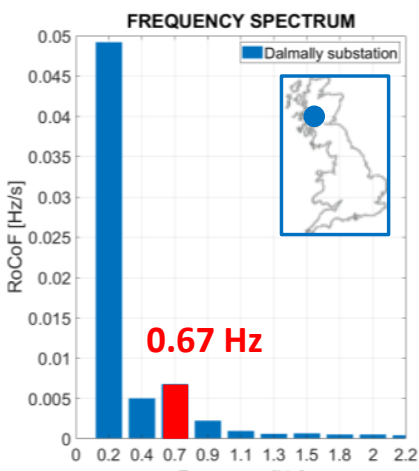

(d)

Figure 8 Comparison of F2P in time and frequency domains at Dalmally substation; PMU data ( $a$ and $b$ ) and OLTA simulation (c and d). 


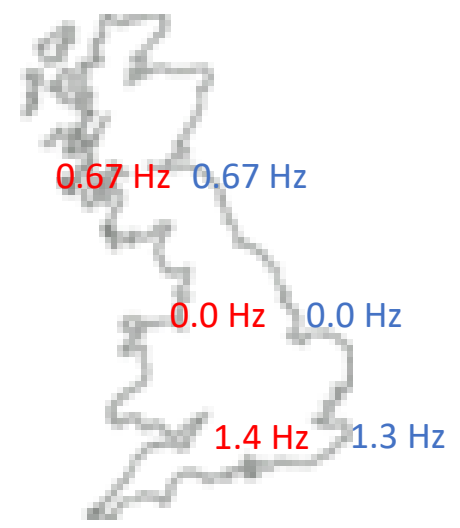

Figure 9 Observed dominant modes from the OLTA simulations (blue) and the recorded PMU data (red).

\subsection{Comparison of Electromagnetic phenomena}

The initial electromagnetic transient measured by the PMUs does not represent any mechanical behaviour of the generators, but rather it originates from a Vector Shift i.e. from the network. Details of this phenomena for the simulation and the PMU data are shown in Figure 10; plotting as ROCOF gives normalized traces, rather than the widely distributed absolute phase angles. The simulated values show initial transient as $1.1 \mathrm{~Hz} / \mathrm{s}$, while the real PMU data $-1.8 \mathrm{~Hz} / \mathrm{s}$. The other difference is in duration; the OLTA simulation shows the transient lasting around $80 \mathrm{~ms}$ while the transient recorded by the PMU lasts for about $200 \mathrm{~ms}$.

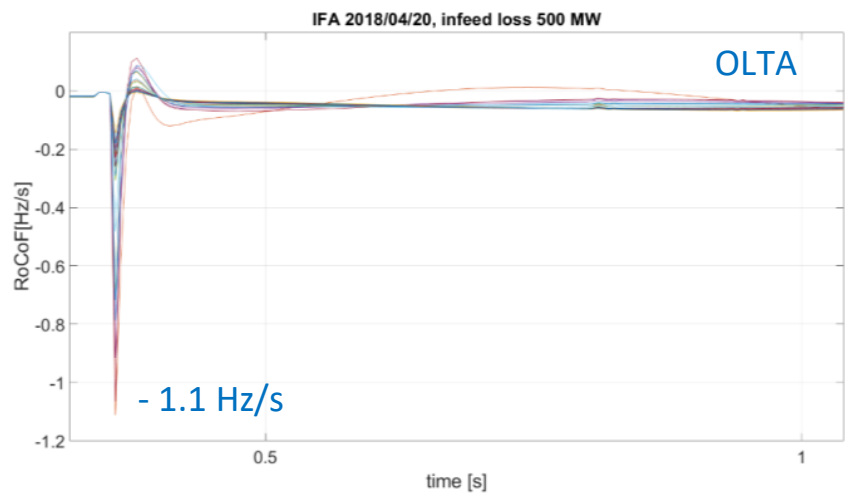

(a)

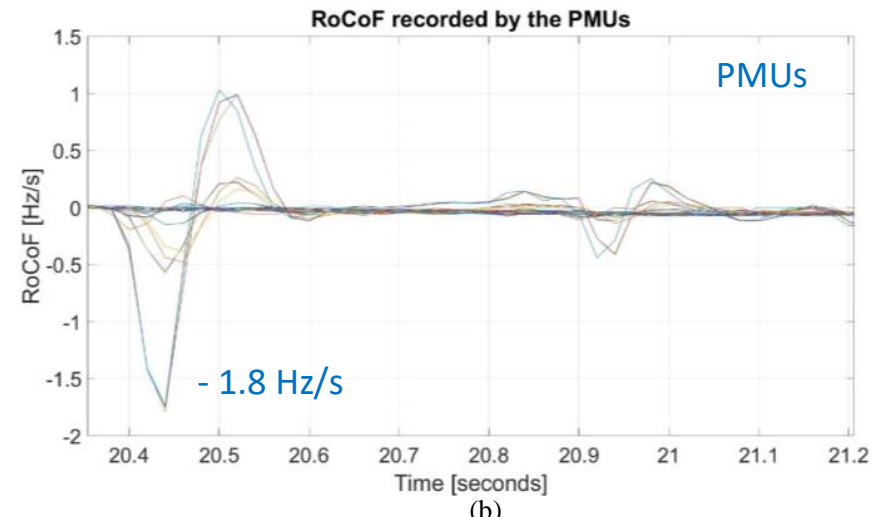

(b)

Figure 10 Comparison of initial transient of study case. (a) OLTA studies of selected buses and (b) PMU data from all PMUs.
The difference between the two may arise from various causes originating either with the simulation model or the PMU measurements. For the simulator, possible reasons could be the sub-transient time constants of the generator models, insufficiently accurate modelling of power electronic in the real system, or modelling of transmission lines as a lumped models instead of using distributed parameters. For the PMUs, results may be influenced by the voltage phasor calculation method, signal processing effects, relatively low sampling frequency of $20 \mathrm{~ms}$, and the involvement of CT/VTs.

\section{Hardware in the Loop studies}

\subsection{Study network}

The validated model of the GB system will be used for Hardware in the Loop (HIL) studies. In order to use this model with the OPAL-RT real-time simulator, however, it needs to be converted from DIgSILENT PowerFactory to ePHASORSIM, the simulation package used by OPAL-RT. This includes the machine and controller models as well as the network. This conversion is still ongoing, and for this paper some initial HIL studies are presented from a standard IEEE 9-bus model adapted to the GB conditions of $50 \mathrm{~Hz}$ and transmission voltage levels, Figure 11. This model was originally developed in PowerFactory and converted to the ePHASORSIM for use with the real time digital simulator OPAL-RT.

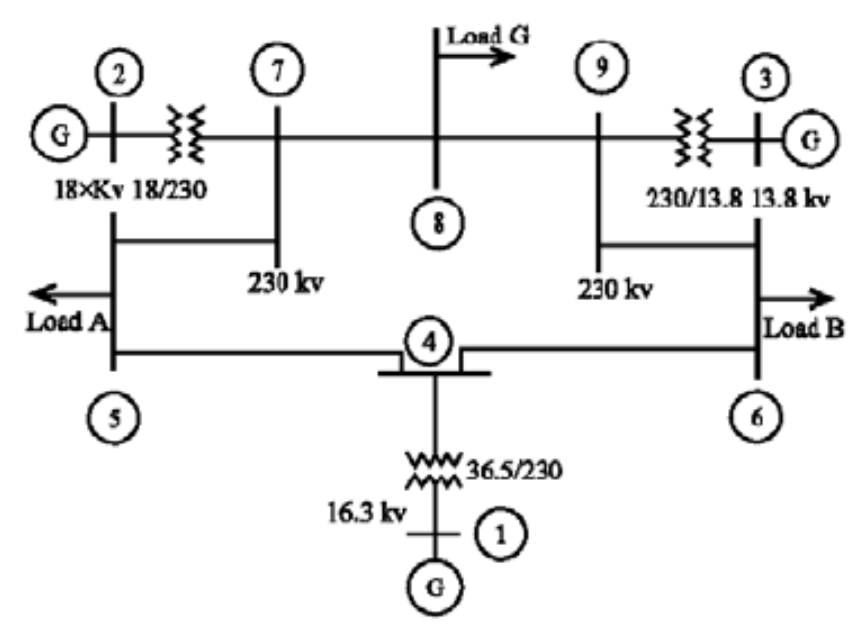

Figure 11 Single line diagram of IEEE 9-bus model. The schematic diagram shows numbers and voltage levels of all busses.

\subsection{Study case}

A disturbance was created by disconnecting generator G1 at various load levels, while total generation and load was kept constant at $300 \mathrm{MW}$. RoCoF and Vector Shift were observed at Bus 3. The voltage of Bus 3 was exported to the analogue output of the simulator and amplified to be used for LoM relay testing. In this case we present a test performed on the IPU/ComAP MainsPro relay. 


\subsection{Results of the simulations}

The results are unexpected at low levels of output for G3 because the frequency rises after tripping, Figure 12. This is caused by a reduction in losses on the network, in turn influenced by the reactive power output of G3 before tripping.

Aside from this anomaly, the HIL studies showed clear borderlines for the disturbance between the relay tripping and not tripping. Relay tripping was consistent around the border-line cases just above and just below the $1 \mathrm{~Hz} / \mathrm{s}$ threshold. For the higher generation loadings, the LoM relay always tripped. Varying the system inertia to 50\% and $200 \%$ of the base case value showed behaviour as expected in the region of interest.

Figure 13 show the laboratory equipment for HIL testing.

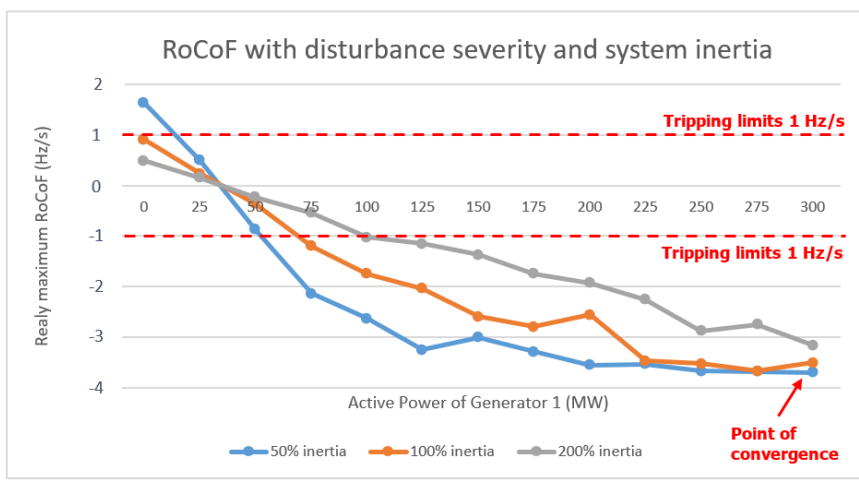

Figure 12 Variation of RoCoF with disturbance severity and system inertia. Maximum relay RoCoF against disconnected generation for a ComAp MainsPro relay.

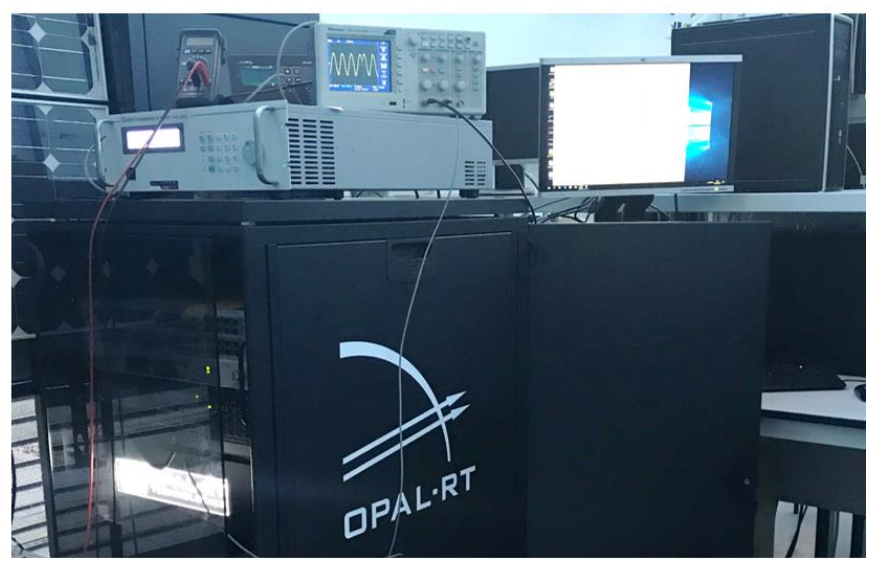

Figure 13 Laboratory set-up for HIL testing.

\section{Conclusion}

In this paper the dynamic behaviour of the full model of the GB transmission system was validated against the PMU data from a real disturbance. Three types of Fast Frequency Phenomena were identified from measurements recorded by the PMUs during the event. The dynamic behaviour of the system (electromechanical and mechanical phenomena) is accurately simulated by the model, but the electromagnetic phenomena is not so well reproduced. Further work is needed to explore the differences between measured and simulated results for Vector Shift effects.

Initial results were also presented for Hardware-in-the-Loop tests on a simplified IEEE 9-bus model, demonstrating the conversion from DIgSILENT PowerFactory to ePHASORSIM and tripping of real Loss of Mains protection relays against simulated events. Future work will involve validation of the reduced GB system model and using both models (full and reduced) for HIL studies and stress testing of the LoM relays.

\section{Acknowledgements}

The research was sponsored by National Grid ESO with Network Innovation Allowance funding from OFGEM (Office of Gas and Electricity Markets)

\section{References}

[1] National Grid, "Future Energy Scenarios," National Grid, Warwick, 2017.

[2] B. U. London, "Investigation \& Modeling of Fast Frequency Phenomena, WP1 - Data Gathering, Visualisation and Investigation," Brunel Univerisity London, 2018.

[3] National Grid Electricity System Operator, "Technical Report on the events of 9 August 2019," National Grid Electricity System Operator, Warwick, 2019.

[4] P. Imris, M. Bradley, G. Taylor, M. Gordon and Y. Li, “Enhanced visualisation of Fast Frequency Phenomena as exhibited in the GB transmission system," in The 54th International Universities Power Engineering Conference, Bucharest, Romania, 2019.

[5] National Grid ESO , "Electricity Ten Year Statement 2018," National Grid ESO, Warwick, November 2018.

[6] S. E. Networks, "Visualisation of Real Time System Dynamics using Enhanced Monitoring (VISOR) -Close Down Report," SP Energy Networks, Blantyre , 2018.

[7] IEEE, "IEEE Standard for Synchrophasor Measurements for Power Systems," IEEE Power \& Energy Society, New York, 2011.

[8] National Grid, "National Grid Transmission: TPCR4 Rollover Response to PPA Energy's review of SO capex," National Grid, Warwick, 2011.

[9] D. Rostom, "Creation of a Reduced Model for the GB network," Nagioanl Grid, Warwick, 2013. 